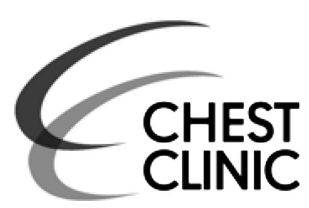
CLINIC

${ }^{1}$ Tuberculosis Section, Public Health England, London, UK ${ }^{2}$ Centre for Lysosomal and Metabolic Diseases, Sophia Children's Hospital, Erasmus MC University Medical Centre, Rotterdam, The Netherlands ${ }^{3}$ Research Department for Infections and Population Health, University College London, London, UK ${ }^{4}$ Centre for Respiratory Medicine, Royal Free London NHS Foundation Trust, University College London, London, UK

\section{Correspondence to} Dr M E Kruijshaar, Centre for Lysosomal and Metabolic Diseases, Sophia Children's Hospital, Erasmus MC University Medical Centre, Dr Molenwaterplein 60, Rotterdam 3000 CB, The Netherlands; m.kruijshaar@erasmusmc.nl

Received 12 January 2013 Revised 29 May 2013 Accepted 6 June 2013 Published Online First 4 July 2013

To cite: Kruijshaar $\mathrm{ME}$, Abubakar I, Stagg HR, et al. Thorax 2013;68:

1172-1174.

\title{
Migration and tuberculosis in the UK: targeting screening for latent infection to those at greatest risk of disease
}

\author{
Michelle E Kruijshaar, ${ }^{1,2}$ Ibrahim Abubakar, ${ }^{1,3}$ Helen R Stagg, ${ }^{1,3}$ Debora Pedrazzoli, ${ }^{1}$ \\ Marc Lipman ${ }^{4}$
}

\section{ABSTRACT}

Active tuberculosis (TB) in the UK predominantly affects the non-UK born, but is generally not manifest at the time of UK entry. Strategies to detect latent TB infection (LTBI) in this population are, therefore, important. To date, targeted screening has focused on TB risk estimates based on the incidence in the country of origin. Using TB incidence in the UK and migration data, we estimated the numbers needed to be tested and treated for LTBI to prevent one case of TB disease. Numbers were the lowest in Somalian and the highest in South African and Filipino migrants, which contrasts with TB rates in these countries. Targeting screening on the basis of incidence in the UK may thus improve effectiveness.

\section{INTRODUCTION}

Unlike the majority of Western countries, the UK has a rising incidence of tuberculosis (TB) which represents an ongoing public health problem. ${ }^{12}$ As almost three-quarters of active $\mathrm{TB}$ cases occur in the non-UK born, TB control needs to focus on this population. ${ }^{2}$ Most non-UK born TB cases are probably infected abroad, with disease resulting from reactivation of asymptomatic latent Mycobacterium tuberculosis infection (LTBI), often many years after entry. ${ }^{13}$ Strategies to detect LTBI in this population are, therefore, important. Screening individuals entering the country for active $\mathrm{TB}$, while important, detects only a small number of cases. ${ }^{1}$

In the USA, migrants are screened for LTBI. Following the 2011 UK NICE guidelines, LTBI testing is now indicated in the UK for new entrants aged 35 or younger from countries where the incidence of active TB is over 40/100 000. ${ }^{4}$ This is despite other health economic modelling suggesting that a cut-off of 150/100 000 may be more appropriate. $^{3}$ The use of such thresholds does not factor in either the actual numbers of new entrants to the UK from different countries or the proportion developing active TB while in the UK. This is important as such information enables particular 'high risk' populations to be more accurately targeted, and hence increase the yield of screening and improve its population impact.

To investigate whether this information can help to better target LTBI screening, we calculated how many UK migrants would need to be tested and treated for LTBI to prevent one active case occurring in the following 5 years. We did this for each of the top 10 countries of birth of UK TB cases, which captures approximately $70 \%$ of all non-UK born TB cases.

\section{METHODS}

Numbers needed to be tested and treated for LTBI using interferon- $\gamma$ release assays (IGRA) were calculated using the number of immigrants aged 35 years or below arriving in 2005/2006. This age cut-off reflects the UK policy of offering LTBI treatment, when needed, to migrants below this age.

We assumed that a proportion of cases aged $\leq 40$ notified with active TB in the 5 years following arrival to the UK could be prevented, depending on test sensitivity and the efficacy of, and adherence to, drug prophylaxis. Numbers tested were obtained as the ratio of the number of immigrants and the number of preventable cases, numbers treated as the number of immigrants testing positive for LTBI divided by the number of preventable cases. We explored the sensitivity of these estimates to migration statistics, test sensitivity and efficacy of/adherence to LTBI treatment using an extreme value approach.

\section{Data sources}

The top 10 countries of birth of non-UK born TB cases reported between 2000 and 2010 to the Enhanced TB Surveillance (ETS) system for England, Wales and Northern Ireland were identified. To calculate preventable cases, we obtained the number of cases aged $\leq 40$ born in these countries who were known to have entered the UK in 2005/2006 and developed disease within 5 years of entry (ie, the cases were reported to the ETS between 2005/2006 and 2009/2010). It was assumed, given the short time frame, that these cases were likely to have been infected pre-entry.

The numbers of migrants arriving in 2005/2006 were obtained from the International Passenger Survey. These data pertain to long-term migrants, that is, people who change their country of usual residence for at least a year, and were kindly provided by the Office for National Statistics.

The proportion of migrants with LTBI $(18 \%-$ $28 \%$, depending on the country) was based on a UK study investigating IGRA positivity in migrants. ${ }^{3}$ IGRA sensitivity was estimated at $84 \%$ $(81 \%-87 \%),{ }^{5}$ efficacy of chemoprophylaxis at $65 \%$ $(50 \%-80 \%)^{3}$ and adherence, based on London 
cohort reviews, at 85\% (70\%-100\%, personal communication, Sarah Anderson, HPA London).

\section{RESULTS}

Table 1 shows the estimated numbers of migrants needed to screen and treat to prevent one TB case for each of the 10 countries of birth (ordered by increasing in-country incidence rate of active TB). Estimated numbers of migrants needed to be tested and treated were the lowest for Somalia and Uganda. For Pakistan, Kenya, Zimbabwe and India, numbers to treat remained below 50, while less than 200 immigrants would need to be tested. Large numbers of South African and Filipino migrants would need to be screened and treated. Low numbers were not necessarily related to a high rate of $\mathrm{TB}$ in the country of origin. For example, while WHO estimates for the TB rate in South Africa were, at almost 1000 per 100 000, higher than in any of the other nine countries, the very high numbers needed to screen and treat for this country indicate a low screening yield.

The table also shows the sensitivity of the results to uncertainty in migration estimates, test sensitivity, and effectiveness of, and adherence to, LTBI treatment. These represent extreme values, that is, when all the estimates either underestimate or overestimate the result. The maximum variation could be substantial, especially for numbers needed to test. Nevertheless, the ranking remained similar, with Somalia and Uganda needing the lowest numbers to be tested and treated, and South Africa and the Philippines the highest.

The number of immigrants reported as coming from Uganda and Zimbabwe were much less reliable as they had a very high standard error, and hence the results from these countries must be interpreted with caution.

\section{DISCUSSION}

The numbers of migrants needing testing and treatment varied substantially across the top 10 countries of birth of UK TB cases and did not follow the rate of $\mathrm{TB}$ in their country of origin. Only 16 Somali migrants would need to be tested for LTBI and four given treatment to prevent one case of active TB developing over the next 5 years. This contrasts with the yield in countries such as South Africa and the Philippines, where much greater numbers of migrants would require testing and treatment to achieve the same impact. Our data use a different approach to current guidance ${ }^{4}$ or recent proposals, ${ }^{3}$ and we believe represents a novel and simple method that adds value to migrant LTBI screening through targeted testing.

The observed differences in the numbers needed to test and treat may result from several factors. The TB rate in the country of origin can vary substantially within the country, and UK migrants may originate from specific geographical areas with rates that are higher or lower than the national average. For example, in South Africa, TB rates in the townships are extremely high, whereas migrants often come from more affluent areas with less TB. Also, differences in demographic and clinical characteristics of migrants from different countries may play a role, such as their socio-economic circumstances before and after arrival to the UK, HIV status and whether their migration to the UK is work-related. Finally, some of the groups within our dataset may already have been tested and treated for LTBI on arrival in the UK. As there is some variability in this practice across the country, it is possible that this may have reduced the numbers of TB cases developing in certain areas (which may be associated with specific migrant groups), but not in others. ${ }^{2}$

Migration data have a high level of uncertainty. Our sensitivity analysis showed that this, together with variability in other estimates, could affect our results substantially. We used an extreme value approach which assumes that all input data would be either underestimated or overestimated at the same time. This, however, is unlikely; and even so, despite larger possible errors, our conclusions remain consistent.

We selected a 5-year time-frame from UK entry to ensure that the majority of the reported TB cases can be assumed to arise from reactivation of LTBI rather than as a result of recent acquisition (either locally or through visiting the country of origin). In fact, approximately $50 \%$ of active $\mathrm{TB}$ in the non-UK born occurs more than 5 years after arrival. Hence, our approach could thus prevent further cases arising after 5 years that have not been captured by our analysis.

Table 1 Number of migrants who would need to be tested and treated for latent infection with Mycobacterium tuberculosis to prevent one active TB case developing in the 5 years following arrival into the UK in 2005/6

\begin{tabular}{|c|c|c|c|c|c|c|}
\hline \multirow{2}{*}{$\begin{array}{l}\text { Country (in-country TB } \\
\text { incident rate per } 100000)^{*}\end{array}$} & \multicolumn{2}{|l|}{$\begin{array}{l}\text { Number of migrants } \\
\leq 35 \text { years }\end{array}$} & \multicolumn{2}{|c|}{$\begin{array}{l}\text { Number of TB cases } \leq 40 \text { years reported } \\
\text { in } 2005 / 2006-2009 / 2010\end{array}$} & \multicolumn{2}{|c|}{ Numbers needed to prevent one case } \\
\hline & Entry in $2005 / 2006 t$ & LTBI¥ & Entry in $2005 / 2006$ & Preventable cases§ & Test (lowest-highest ๆ) & Treat (lowest-highest $\uparrow$ ) \\
\hline Nigeria (133) & 15000 & 4,200 & 127 & 59 & $254(127-523)$ & $71(36-146)$ \\
\hline India (185) & 92000 & 18400 & 1073 & 498 & $185(109-338)$ & $37(20-61)$ \\
\hline Uganda $(209)^{* *}$ & 1000 & 280 & 25 & 12 & $83(25-224)$ & $23(7-63)$ \\
\hline Bangladesh (225) & 18000 & 3600 & 170 & 79 & $228(129-434)$ & $46(26-87)$ \\
\hline Pakistan (231) & 42000 & 8400 & 532 & 247 & $170(85-349)$ & $34(17-70)$ \\
\hline Philippines (275) & 16000 & 2880 & 69 & 32 & $500(177-1176)$ & $90(32-212)$ \\
\hline Somalia (286) & 3000 & 840 & 406 & 188 & $16(6-37)$ & $4(1-7)$ \\
\hline Kenya (298) & 3000 & 840 & 39 & 18 & $167(63-390)$ & $47(13-78)$ \\
\hline Zimbabwe $(633)^{* *}$ & 7000 & 1960 & 87 & 40 & 175 (38-467) & 49 (11-131) \\
\hline South Africa (981) & 41000 & 11480 & 72 & 33 & $1242(643-2492)$ & 348 (180-698) \\
\hline
\end{tabular}

*Incountry TB rate estimated by WHO (Global tuberculosis control: WHO report 2011).

tNumber of long-term migrants rounded to the nearest thousand, estimated from the International Passenger Survey. Source: Office for National Statistics.

$\ddagger$ \#ased on estimates of interferon- $\gamma$ release assays positivity by world region from Pareek et al. ${ }^{3}$

§Preventable cases-adjusting the number of TB cases for test sensitivity plus preventive therapy efficacy and adherence.

IHighest and lowest estimates using the upper and lower range of sensitivity, efficacy and adherence estimates and the $95 \% \mathrm{Cl}$ of the number of migrants.

**Data from the International Passenger Survey are thought to be unreliable (standard error $>35 \%$ ).

LTBI, latent tuberculosis infection; TB, tuberculosis. 
The estimates presented do not take into account the effects of TB drug resistance, HIV co-infection, acceptance of chemoprophylaxis, changes over time in migration and costs of screening and treatment. While drug resistance and HIV co-infection will reduce the efficacy of chemoprophylaxis (and the latter also IGRA sensitivity), most people with LTBI will be drug sensitive and HIV negative. Thus, the effect on our estimates will be small. Additionally, the results of our sensitivity analysis show that our conclusion is robust against such uncertainty.

A key next step is to improve migration data, for example, using other immigrant information sources. This would enable cost-effectiveness analysis to be accurately performed which in turn will provide useful evidence when evaluating targeted LTBI screening.

\section{CONCLUSIONS}

TB incidence in the country of origin alone does not accurately predict the number of cases subsequently arising in the UK or the yield from LTBI testing of immigrants. LTBI testing could be improved by targeting specific populations, which we believe will result in increased yield and enhanced value and cost effectiveness.
Contributors MEK played a role in the design of the study and interpretation of the results. She carried out the analyses and drafted the manuscript. IA and ML conceived of the idea for the study and were involved in the design, interpretation of results and writing of the manuscript. HRS and DP contributed to the analyses and interpretation of the data, and critically reviewed the paper. ML is the guarantor of the study. All authors read and approved the final manuscript.

Competing interests None.

Ethics approval The PHE has Health Research Authority approval to hold and analyse national surveillance data for public health purposes under Section 251 of the NHS Act 2006

Provenance and peer review Not commissioned; externally peer reviewed.

\section{REFERENCES}

1 Abubakar I, Lipman M, Anderson C, et al. Tuberculosis in the UK-time to regain control. BMJ 2011;343:d4281.

2 Pedrazzoli D, Kruijshaar M, Anderson L, et al. Tuberculosis in the UK: 2011 report. London: Health Protection Agency, 2011.

3 Pareek M, Watson JP, Ormerod LP, et al. Screening of immigrants in the UK for imported latent tuberculosis: a multicentre cohort study and cost-effectiveness analysis. Lancet Infect Dis 2011;11:435-44.

4 Clinical diagnosis and management of tuberculosis, and measures for its prevention and control. London: National Institute for Health and Clinical Excellence, 2011. http://publications.nice.org.uk/tuberculosis-cg117/guidance

5 Diel R, Loddenkemper R, Nienhaus A. Evidence-based comparison of commercial interferon-gamma release assays for detecting active TB: a metaanalysis. Chest 2010;137:952-68. 